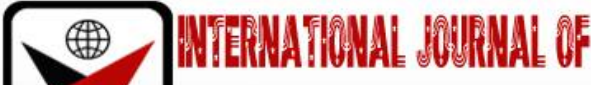 \\ בितिक
}

ISSN 2278 - 0211 (Online)

\section{Mapping HIV/AIDS High Risk and Vulnerable People in Communities' Most at Risk Population}

\begin{tabular}{c} 
Doreen Nekesa Busolo \\
Ph.D. Candidate, Centre for Communication and Social Change, \\
School of Communication \& Arts, University of Queensland, Australia \\
Samuel Ngigi \\
Lecturer, School of Journalism and Mass Communication, University of Nairobi, Kenya \\
\hline
\end{tabular}

\begin{abstract}
:
Over one billion USD is the total financing towards HIV burden in Kenya according to the USAIDS (2018) report. The finance from the government, private sector, and international agencies are directed towards the fight of HIV in Kenya. The HIV key population identified in Kenya include sex workers, Men who have Sex with Men(MSM), People Who Inject Drugs (PWID). On the other hand, transgender and people in prison are not listed among the key population, however, their behaviours put them at risk of contracting HIV.Ninety-five percent of sex workers and ninety-four percent of PWID know their HIV status. It is important to note that the MSM population regarding the same is not known (UNAIDS 2018). This is an indication of criminalization of certain behaviours by law exposing them to high-risk. For instance, the number of transgender people in Kenya is not known posing a challenge in planning and designing HIV intervention measures for this group. Also, the figure of transgender people in Kenya is not known due to fear of rejection by the society where many cultures do not recognise transgender. A good number of the key population know their HIV status and emphasis should be on how to prevent the spread of the virus and those not infected to use protection as they engage in risky behaviours.
\end{abstract}

Keywords: Mapping, HIV/AIDS high risk, vulnerable Communities', most at risk population, Men who have sex with men (MSM), People who inject drugs (PWID), transgender

\section{Introduction}

The general concern for HIV/AIDS is with people whose behaviour puts them at increased risk of infection (UNAID and WHO 2010). Mapping helps to identify populations at higher risk of HIV infection thus promoting HIV intervention programs in the affected areas. Safety measures can be achieved by identifying key population at-high-risk of new infections. This will enhance good planning by the government and other agencies to provide health-related services to the affected group.

National AIDS Control Council (NACC) 2009 revealed that a third of all new HIV infections in Kenya in 2008 were among some of the most at-risk populations. This is emphasized in the report by Population Council (2011) which revealed that Kenya's HIV epidemic affects most of its general population, however, people who inject drugs, men who have sex with men and women, and sex workers are still more vulnerable to infection. The government needs to identify the key population to help curb new HIV infection.

Kenya is the fourth-largest HIV epidemic in the world with 1.6 million people living with HIV in 2016 (Avert 2017). The report further revealed that $65 \%$ of all new infections occurred in nine counties mainly on the west coast of Kenya. However, risk behaviors change over time due to economic activities, politics, seasonal migration, the introduction of laws prohibiting risk behaviors, outreach programs to change risk behaviors among others. Such changes can either prevent or increase the number of new infections.

On the other hand, the risk of getting HIV varies depending on the type of exposure or behavior (PMC 2016). Shocking findings by PMC (2016) revealed that high-risk population members who were HIV infected were generally unaware of their HIV infection. Although they actively engage in unsafe risk behaviors, such as inconsistent condom uses and high numbers of sexual partners indicating new infections. There have been several awareness campaigns by the government and other concerned agencies regarding the HIV epidemic. The point of concern is whether the message reaches the target group or is it a matter of ignorance.

The current population of people living with HIV around the globe is about thirty-eight million people (37.9 M) according to WHO (2018) report. One point seven million were new HIV infections. The sad news is that most affected region is Africa where one in every twenty-five adults are living with HIV. In 2017, Kenya new HIV infection was one point five million people (UNAIDS 2018). This figure is still high considering that health burdens are a societal burden affecting an individual as well as the society. 
Different methods have been applied by different countries in mapping HIV/AIDS high-risk and vulnerable population in the community. For instance, ethnographic mapping, broad mapping and site assessment, Participatory site assessment (PSA), Social network analysis (SNA), Community-led situational needs assessment (CLASS), mapping, enumeration and needs assessment (MENA), Micro-planning among others.

However according to (JAIDS 2018), the challenges such as some of the behaviors that place key populations at increased risk of acquiring and transmitting HIV are illegal in Kenya, the number of some people involved in such activities is unknown. For instance, PWID performs the act in secluded environments, due to fear of criminalization and stigmatization. National mapping of the key population in 2012 revealed 133,675 FSWs, 18,460 MSM and 18,327 PWID (Musyoki et al 2018). There is a need for another mapping to include adolescent, youths, transgender as well as people in prison.

On the other hand, behavior such as low condom uses with regular non-paying partners, drug overdoses among PWID among others remains a substantial health risk to key populations (Musyoki et al 2018). Emphasis on behavior change as an intervention measure should be given priority considering that new key population groups are emerging increasing the number of key population groups. This can be achieved through well-designed programs in response to key population-specific needs.

\subsection{Background}

Female Sex Workers (FSW), Men who have Sex with Men (MSM), and People Who Inject Drugs (PWID), have the highest risk of contracting and transmitting HIV in Kenya. They also have the least access to prevention, care, and treatment services due to their behaviorsthat are often stigmatized, and even criminalized (NACC 2016).

HIV \& AIDS epidemic in Kenya is socially and geographically heterogeneous with certain counties and populations having higher HIV prevalence and incidence than others (Musyoki et al 2018). JAIDS (2018) report identified the HIV epidemic concentration in five counties namely Homabay, Kisumu, Migori, Siaya, and Nairobi with $50 \%$ of the unmet antiretroviral treatment need.

Musyoki et al (2018) report further revealed that the likelihood of acquiring HIV among people who inject drugs (PWID) is twenty-four times higher than adults in the general population, ten times higher among female sex workers (FSWs), and twenty-four times higher among men who have sex with men (MSM). There significant higher HIV prevalence among people who inject drugs than in the general population in Nairobi and Mombasa (Mbete, Nougier and Bridge 2013). NACC (2016) report indicated that Women in Kenya are more vulnerable to HIV infections compared to Kenyan men, with the national HIV prevalence at 7.0 per cent for women and 4.7 per cent for men. Musyoki et al (2018)findings confirm that one-sixth of female injecting drug user also sell sex to paying clients thus increase overlapping dimensions of risk and vulnerability for HIV infection.

Narcotic Drugs and Psychotropic Substances Control Act, prohibits any Kenyan to smoke, inhale, sniff or use any narcotic drug. The law further criminalizes any person found where drug use is happening, preparation or sale of drugs in the premises; and possession of pipes or other equipment for use in connection with drugs, including needles and syringes (Mbete, Nougier and Bridge 2013). This put PWID at more risk of new HIV infection through needle sharing.

On the other hand, MSM lack social support from the community due to their unacceptable behavior in society. Fear of marginalization has affected the uptakeof HIV prevention measures among MSM and any measure towards this course is done in total discreet. This means that not all MSM is reached thus affecting the implementation of programs for HIV prevention among the key population.

Geographical mapping of key population concentration is important for the nation in terms of planning towards health services for the key population. On the other hand, social norms towards certain behaviors in the community must be addressed to enhance reach to all individuals in hiding for fear of discrimination.

Mapping has been used in public health to understand the geographic characteristics and distribution of diseases. However, this must be conducted in a way that respects the needs and safety of people involved (Musyoki et al 2018). Also, how and when is important considering that methods used should conform to the culture or norms of the community to ensure that no victimization of the affected individuals arises.

Key population-based in different geographical places have different behaviors influenced by different factors as will be addressed later. Behavioral intervention focusing on individual as well as the society should be a continuous process to fight HIV new infections. Also, it is important to map the affected areas to help in planning as well as designing appropriate methods to be used in the intervention process.

\subsection{Mapping Hiv/Aids High Risk and Vulnerable Populations in Communities}

Mapping is an operation that associates each element of a given set (the domain) with one or more elements of a second set. Mapping is used in health programs to inform the design and implementation of programs that will help to address specific patterns of structural and behavior risk that increases HIV prevalence. There are geographical areas or locations with evidence of the high prevalence of HIV or behaviors that put people at risk for acquiring HIV infection. For instance, the HIV epidemic in Kenya is concentrated in five counties namely Homabay, Kisumu Migori, Siaya, and Nairobi (JAIDS 2018).

The common groups of the population at high risk of contracting HIV \& AIDS include sex workers, men who have sex with men (MSM) and people who inject drugs (PWID). The groups at high risk may be more than what is mentioned above. For instance, truck drivers, migrant, youths, fishermen and any other person with behaviors that place him/her at risk of contracting HIV; like the exchange of sex for services rendered or goods received expose people to HIV infection. 
These people are found in the society and they are vulnerable to HIV infection due to different factors that place them at risk of contracting HIV.

NACC (2016) report revealed that women in Kenya are more vulnerable to HIV infections compared to men, with HIV prevalence of 7.0 per cent for women and 4.7 per cent for men as per the 2015 HIV Estimate report. Youth experiencing adolescent according to Avert (2017)engages in unprotected sex behavior which is the most common route of HIV infection among young people followed by the sharing of infected needles. This is due to the lack of correct knowledge about HIV and how to prevent it or being forced to have unprotected sex or to inject drugs. The report further states that half of the 15 to 19-year-olds who are living with HIV in the world live in just six countries: South Africa, Nigeria, Kenya, India, Mozambique and Tanzania.

In 2014, the government of Kenya through the Ministry of Health invested in prevention of HIV new infections at the County level by using evidence-based and strategic guidelines to enhance comprehensive and population-based approach that includes biomedical, behavioral and structural HIV programs targeting various populations at higher risk. In the same year, the Kenyan government established a geographical approach to help prevent new infections. The geographical approach was County-based where 47 Counties were divided into three clusters i.e. high, medium and low in HIV incidents (Ministry of Health 2014)

This approach was arrived at to help address structural factors and barriers that increase key population vulnerability. However, the worry of new infections among the key population continues especially the groups that are culturally not accepted, like Men who have sex with Men (MSM). Cultural barrier is making it difficult for the government and other agencies to fight new infections among gay community and lesbians. People who inject drugs, on the other hand, perform the illegal act secretly to avoid being published by the law. Also, sex workers prefer their behavior to remain private for them to remain relevant to the community.

The three aspects challenging the fight for HIV new infections among the people at high-risk include socialcultural barriers, legal barriers and behavior change barriers. The three must be carefully addressed. This means that the government, stakeholders, the community and other agencies to fight HIV new infections to work together to achieve this goal. The community-based organizations, the community leaders, the key population representatives, the government through the ministry of health and the legislature must address this issue as a team working towards achieving a common goal.

Community-based organizations are good in providing access to the community as well as relevant information. Groups such as self-help groups comprise of people with similar interests. Group members share similar condition and being together as a group is an assurance that one is not alone. Individuals are empowered through the member's experience. The collective thinking and problem-solving in the group have enabled people to learn how others manage their situations. It also helps the group to find solutions suitable for their collective situation.

The interaction between peer groups of people at high-risk is important in preventing HIV new infections. The sense of belonging is felt at this point. This may lead to breaking the barriers of misunderstanding, mistrust and intimidation. Also, outreach programs to vulnerable groups and key populations can help transcend barriers. Through outreach programs, the community will become aware of key population groups living among them and find a way of assisting them.

Mapping of high-risk behavior targets local groups who are members of the society. However, foreigners residing in the community are not considered risk groups yet they are also exposed to risk behaviors. For instance, drug use in Malindi is common among foreigners and local youths and sharing of the needle is one way of transmitting diseases. Distribution of syringe and condom normally targets the local members of the society.

\subsection{HIV Special Populations in Kenya}

According to USAID's Gap Report,(2014) HIV prevalence is estimated to be twenty eight times higher among people who inject drugs, twelve times higher among sex workers, nineteen times higher among gay men and other men who have sex with men and up to forty nine times higher among transgender women than among the rest of the adult population. Majority of African society does not approve the behavior and such an act is illegal. People involved in homosexuality are considered unacceptable.

The Kenya AIDS Strategic Framework 2014/15-2018/19 (KASF) prioritizes the key populations of Female Sex Workers (FSWs), Men who have Sex with Men (MSM) and People Who Inject Drugs (PWID). The number one strategy KASF is to reduce annual new HIV infections among adults by seventy-five percent and reduce HIV transmission rates from mother to child from fourteen percent to less than five percent.

The government is more concerned with the named groups above; however, more groups are at high risk of acquiring and spreading HIV. One of the forgotten groups is the transgender group. It is very dangerous to ignore the transgender group which in reality is at most risk of HIV infections. The identity issues surrounding the transgender group forms the basis of stigmatization. Cultural norms prohibiting transgender persons from seeking health services is a major threat to HIV prevention. The information about HIV infection among transgender people in Kenya is not documented.

The strategic plan in place may not achieve the desired outcomes if some groups that are emerging for instance transgender group are not put into consideration as a priority group. Such risk groups are engaging in risk behaviorism confidentiality to avoid rejection from the community. Some want to fit in the society and they end up marrying or getting married to fool the society however they continue with their other life in secrecy.

Men who have Sex with Men are criminalized in Kenya. Fear of isolation has hindered most of them from accessing health services. Due to this, HIV infected members of this group lack treatment because they fear to reveal their status to other people, not even their family members. They suffer in silence together with their partners due to fear. 
Similarly, people who inject drugs fear to face the law. They continue to inject drugs, despite warnings and information about risks related to injecting drugs. Sharing of the needle is a risk by itself and the impact of drugs is also another risk that can lead to multiple risk behaviors.

PWID are most-at-risk of contracting HIV either by using a contaminated needle or engaging in risky sexual behavior under the influence of drugs. Harm reduction measures such as needle exchange programs have been proven to reduce the transmission of blood borne pathogens such as HIV, HBV, and HCV (Mbete, Nougier, \&Bridge 2013). However, such programs may not reach all PWID because not all of them have come out to seek help.

UNAID (2014) report revealed that HIV prevalence among MSM in Kenya is almost three times that among the general population. The law prohibits this act and anyone found guilty is entitled to 14 years sentence. The barriers in place have been and still the existing gap preventing good planning and designing of relevant intervention measures towards HIV prevention thus the occurrence of new infections among the high-risk groups.

Ministry of Health and other agencies such as the National Empowerment Network for Persons Living with HIV and AIDS in Kenya (NEPHAK), The Kenya Sex Workers Alliance (KESWA), The Bar Hostess Empowerment and Support Programme (BHESP), and the Global Network of People Living with HIV (GNP+) have been towards the same goal, preventing the spread of HIV virus. The above organizations in (2015) on female sex workers in Nairobi revealed that around one-third were living with HIV.

Female sex workers are among the key population group identified in Kenya AIDS Strategic Framework 2014/15 - 2018/19. Female sex workers have been the victim of not only getting infected with HIV and STI's but also violence. Societal discrimination has impacted their social life negatively thus preventing them from seeking help to avoid shame. The Ministry of Health (2018) report identified sex work as consensual sex between adults, can take many forms, and varies between and within communities.

These women are at risk of getting infections because sometimes they get involved in other behaviours like alcohol and drug abuse that puts them at high-risk. When they are intoxicated, they cannot negotiate the use of protections with their client. The community that is condemning these women making them inferior and not being able to seek help is at risk as well. There are high chances that the virus will be transferred to their customers who are also members of the community.

Addressing female sex workers issues is a challenge by itself. The law prohibits the act, the society stereotypes sex workers, and the young girls are joining this way of life due to various reasons. The latter is very dangerous because the young girls have been forced into this kind of life-denying them from enjoying their childhood. Their future is affected and their dream shattered. The young female sex workers are likely to transmit the virus to more people and especially young men of their age.

Report by the Ministry of Health (2018) National Implementation Guidelines for HIV and STI Programming among Young Key Populations revealed that there are varying reasons why young people sell sex. Some of the reasons were: they select sex work as an occupational choice to escape poverty, meet financial obligations, or support their families, and/or because gender inequality, discrimination, or bullying limited their access to education, and by extension, their earning capacity.

Youths are among the vulnerable group that is at high risk of acquiring HIV. Peer pressure among youth puts them at risk of contracting HIV. The UNAIDS (2018) report revealed that over fifty per cent of new HIV infections in Kenya in 2015 occurred among the youths aged fifteen to twenty-four. This group comprises of adolescent young people with little knowledge about HIV. There are several contributing factors other than peer pressure that has led to the exposure of youths to HIV infections.

Adolescent stage in development stages comprises of challenging factors that put young people at risk. At this stage of transition to adulthood, young people try to discover and the same time understand who they are and if they do not get proper guidance, they are likely to be misled into unhealthy behaviors that may put them at risk. For instance, they are likely to engage in unprotected sex, drug abuse, alcoholism etc. 'The increasing rate of HIV infection among young people including the incorrect perception of HIV risk; and having unprotected sexual intercourse under influence of alcohol or drugs' (UNAIDS 2018).

Women are also at high risk of contracting HIV. Considering the gender perception where women have no voice to demand for their rights puts them at risk. For instance, young girls are married off to old men some older than their parents. In this kind of marriage, the young girl cannot speak for herself even although her rights are violated. A survey by Gitau, et. al. (2016) on child marriage, teenage pregnancy and female genital mutilation/cutting in Kenya revealed that about 23 per cent of the participants were married off and forty-nine percent were below eighteen years at the time of marriage. This includes nineteen percent who were married before they celebrated their fourteenth birthday.

Different aspects are contributing to HIV new infections that need to be addressed. Dealing with the root cause of other factors leading to new HIV infections must be addressed with urgency. The society, government, agencies and people of goodwill must work together to address this issue. The gender inequality should not be permitted to show its masculinity at this point. The young female sex workers can be accommodated to schools and allow them to build their future. Media campaigns and other means must be used to reach out to the community as well as sex workers to help address the problem. Behaviour change campaign mechanism should be a continuous approach and the more repetitive the information, the greater the impact on behaviour change.

\subsection{High-Risk Behaviors for HIV}

There are various schools of thought regarding human behavior and how some people behave the way they behave. Some behaviors are genetically associated while others are socially influenced. For this study, High-risk behaviors 
concerning HIV infections are socially related. The forces underlying human behaviors that put them at high risk of contracting HIV in most cases are environmentally associated. Practicing unprotected sex, multiple sex partners, engaging into extramarital sex, engaging into sex while under influence of drugs/alcohol, peer pressure, lack of knowledge about HIV and AIDS etc.; are some of the common behavior's that can result into HIV infection. Biglan et al (1990) confirm that having sex with a person of unknown status is a risk.

The lifestyle that people choose to live can also expose them to contracting HIV. The element of decision-making in terms of ability as well as knowledge to consciously decide your life as an individual is very important. A good example to help us understand is wife inheritance. Although one will argue that wife inheritance is not a behavioryes, it is not, however, is a cultural practice that perpetuates the spread of HIV among the communities that practice the above culture. In such a situation a widow is forced into sexual engagement with the inheritor.

NCBI (2014) affirms this when they state that widows must balance limiting their risk for HIV infection with meeting cultural expectations. At this point, both the widow and the inheritor are at risk of contracting HIV. On the other hand, this encourages multiple sex engagement especially when the inheritor is married. In a situation where one person in this cycle is HIV positive then the spread to all involved is assured. Also, some key population exchange sex for the drug for instance due to lack of finance.

Lifestyle choices as mentioned above is an individual decision but sometimes the influence of others in the environment we live in can affect the way we make the decision. Auerbach, Wypijewska and Brodie (1994) confirm that multiple factors at the individual as well as environmental level determine our behavior. Also, these factors influence or are influenced by another. A good example is when excessive consumption of alcohol influences engaging in unprotected sex.

The sexual activity in terms of the number and the nature of sex partners determine the outcome of sexual reproductive health (Resenberg et al 2019). The researchers in this study identified structural factors such as socioeconomic as well as unequal gender norms that expose especially women to HIV infection. Both male and female involved in multiple sex activities expose themselves to high risk considering that there are high chances of infection within the network. For instance, one person infected with HIV within the network exposes the majority within the same network to acquiring the virus.

Such behavior is common in specific settings within the communities we live in. For instance, Auerbach, Wypijewska and Brodie (1994) affirm that sex and drug use behaviors are common in specific settings. In Kenya, such behavior is common in social gathering such are organized events, bars, massage parlor, house party, funeral ceremonies in the rural areas etc. There is plenty of drinks in such gathering and some people are lured into risky sexual behaviors due to alcohol influence.

The sexual activities in the massage parlor are more organized in the sense that the privacy is given priority and the public could not easily know what happens there. Kibicho (2009) revealed that 'massage parlors are quasi-legal fronts for sex trade because massage is legal but sexual activities which take place in these parlors are illegal.' Commercial sex activities in such place are professionally organized where the adverts are put up in newspaper and the availability of social media has made is more accessible. The public is fooled by the disguised adverts preventing them from suspecting any risk behaviors in the parlors.

The environment in these places encourages risk behavior in the sense that commercial sex workers are trending online in the name of socialites. The mainstream media is also fooling the society by blindly covering the so-called socialites but not revealing the truth to the public. The spread of HIV at this level cuts across the board, those with knowledge about HIV find themselves in such a situation due to the choices they make. People with no knowledge get infected because they have no idea. This leads us to the next point where social-economic factors also contribute to HIV infections.

Social-economic factors can also be experienced when the individual sell sex to earn income. This is more among female both adult and young girls who engage in such activities due to different circumstances surrounding their lives. Human behavior according to Mayer and Pizer (2009), everywhere in the world is modified by differing local demographics, geography, culture, economics and politics. Similar thoughts were raised by Auerbach, Wypijewska and Brodie (1994) when they stated that 'social networks and the norms that govern the behavior of their members exist within communities including those defined by culture.'

People at high-risk belongs to the community and they are members of the society where we all belong. WHO (2004), revealed that some behavioral risks are realized due to the absence of protective factors. The impact of risk behavior affects all of us in one way or another. Meaning that the fight against HIV new infections, is a global fight that requires international cooperation. Mayer and Pizer (2009), agrees that HIV can easily spread across the planet through international activities like peacekeeping as well as sex tourism.

\subsection{Management of Special Groups}

Special groups identified in the key population group section lives among us. These special groups were identified due to their behavior that put them at risk of acquiring and/or spreading HIV. To prevent new HIV infections, there is a need to manage special groups. Researchers, governments, and concerned agencies have been working tirelessly towards HIV prevention around the globe. Various strategies have been developed and can be applied in a different environment as well as among specific group through targeted intervention measures.

Behavior change strategic intervention is important at this point having learnt that high-risk behaviors are major contributors of new HIV infections. Approaches towards preventing new HIV infections require imparting knowledge to the society about HIV. Ngigi and Busolo (2018), affirms that there is an increase of knowledge, exchange of ideas, reduction 
of stigma as well as attitude change through behavior change programs. An educated society is well placed to fight the virus by applying correct measures in place. Considering the argument by Marlatt, Larimer and Witkiewitz (2012), harm reduction approach is the most effective way of preventing new HIV infections when integrated into the community. They suggested the effective integration of grassroots activist with global public health to ensure continued progress in the fight of HIV new infections in the community.

In Kenya, the public opinion leaders in the community, Community Health Volunteer (CHV's) together with the Ministry of Health should work together through organized coordination to help prevent HIV new infections. Door to door as well as media campaigns should be a continuous process to emphasize the message that is being replied to the audience. In this case the key population. Preventing radical behaviors at the individual level as well as group level is one measure that will help the fight against new HIV infections.

Using communication to address certain behaviors will help prevent multiple infections resulting from different high-risk behaviors. For instance, person injecting drugs has high chances of acquiring HIV if he/she shares a needle with others. The same person is likely to transmit the virus to his/her sex partner and chain is likely to continue. Public health campaigns targeting specific groups sensitize the community by creating awareness regarding HIV. Ratzan (1993) affirms that applying strategic health communication is the best measure considering that there is no vaccine for HIV.

Although the above measure seems to be a good approach to fight HIV new infections, it is very difficult to change behavior. Factors such as diversity of the key population in terms of culture as well as behavior according to Ratzan (1993), must be considered when designing health communication campaign messages. In Kenya transgender as well as people in prison are not among high-risk behavior group although they exist. There is a risk of inmates when they return to the community especially if they get infected with HIV while in prison. Education on safe sex in prison as well as drug injection should be given importance. The fight against new HIV infections mainly focuses on MSM, sex workers and people who inject drugs. To fight HIV, the government should find a way to address transgender and people in prison.

Managing special groups is a challenge that requires diverse approaches to prevent HIV new infection. The major challenge in most parts of the world is the implementation part of some of the intervention measures in place. In Kenya, the religious beliefs that prohibit their congregation from seeking health services is a threat to the fight of HIV. This means that there is no data on HIV/AIDS from this group.

The transgender people neither their behavior is not recognized by the society nor no open discussion concerning this group is welcomed. It is a taboo in the community to talk about such people since most communities' beliefs in male and female gender and transgender are people seeking attention. Some people blame the transgender issue on poor parenting that has allowed gender transition. Since there is little information concerning this group, there is little disclosure for fear of rejection.

The behavioral acts are secrets they keep among themselves exposing them to HIV infections. Some masquerade as male or female to be accepted in society. It is difficult to address HIV infections among this group. At the community level, the transgender expression through behavior due to the sense of feeling of being another gender other than what you were identified with at birth does not count.

\subsection{Community Social Support Structures}

Care and support for HIV victims in our communities is an approach that should be encouraged in society. Ramirez-Valles (2002) confirm that community involvement plays a central role in the fight against HIV/AIDS. To help us understand better what the community is and how the community can help fight the spread of HIV, UNAIDS (2015) identified community system as community-based structures and mechanisms used by the communities to enable community members and CBO's and groups to interact, coordinate and deliver. A community is Kenya is identified by geographical settings, people speaking the same language, sharing cultures, social practices, beliefs and values.

Engaging the community in the fight against HIV/AIDS empowers the community with education about HIV. The community members through education become conscious about the HIV risks. Also, education enables the community members to effect change within the community by intervening measures culturally appropriate (Ramerez-Valles 2002). The government of Australia, for instance, has encouraged community-based HIV organizations to help fight HIV/AIDS. The organizations were established from the affected communities (AFAO and Australia's State and Territory AIDS Council 2016). More emphasis was presented by Ferrand and Kranzer (2014) when they revealed that community-based counselling and testing helps in early diagnosis thus permitting early intervention. Individuals found to be HIV positive are counseled advised to leave positive life by avoiding sexual risk behaviors.

Community support to HIV patient is the best way to reduce stigma. It also influences the victim to accept their status considering that the community will not judge them. The victim seeks emotional support from members of the community. Support from the family and friends may reduce stress and help the victim to cope with his/her condition. Most of the victims are in denial, and even ashamed when they learn about their status and the community/family on the other hand sometimes blames them mounting pressure on the victim.

Stigma affects the social life of HIV patients worsening their condition. Allden and Murakami (2015), encourages home-based care as well as physiological works to help counter misinformation and discrimination of HIV patients. It is important for both the community and HIV patients especially at the time they learn about their status to get support from the community since the majority experience overwhelming distress.

Education and information remain key in the fight against HIV new infections. The effectively mobilized communities towards HIV/AIDS are well empowered to prevent the virus from spreading. Communality working together with all stakeholders such as government, religious groups, traditionalist, NGO's media and the Ministry of education 
should be involved in designing HIV campaigns. This will help create an impact on the targeted group considering that key population groups engage in different behaviors exposing them to HIV infection.

Also working together will enhance referrals where victims can easily be traced and included in health programs available. This will provide data of the HIV patients in the country thus enhancing good planning for the government and the service provider. Community outreach encourages community members to know their status by testing and also promotes awareness through education. Intervention measures such as home visits especially for HIV victims ensure correct procedures are followed as advised by the health service provider.

Although knowing HIV status has been termed as one way of preventing HIV infections, most studies confirm that HIV testing among men is low in many parts of the world. For instance, DiCarlo et. al. (2014), Bowser, Quimby and Singer (2007), Katirayi, et.al. (2017) etc. revealed that many factors such as masculinity, culture, and structural barriers such as occupation where men claim to be busy are some of the reasons preventing men from testing. On the other hand, fear has been identified as a barrier to HIV testing 'for men, it is better not to know one's status than to deal with the emotional consequences of HIV-positive test results' (Bowser, Quimby and Singer 2007).

\section{Conclusion}

The fight against new HIV infections should not be left to the government and concerned agencies alone. This war concerns everyone as an individual as well as the community. The victims belong to society and their health problems affect society in many aspects that should not be ignored. For instance, the inability to engage in productive activities due to poor health is not only a burden to immediate family members but to the nation at large. Advocacy for behavior change should be given priority to prevent new HIV infections.

Individual acceptance is a very important aspect to fight HIV new infections. Infected persons should be in the front line to help fight the spread of HIV. It is their responsibility to take up the correct measures to prevent the spread of HIV. Imparting knowledge about how HIV is transmitted and how to prevent it from spreading should be a continuous area of discussion across all type of people regardless of their status. Among the key population identified in Kenya, seventy-three percent of sex workers, around sixty-three percent of MSM and above sixty-seven percent of PWID are on antiretroviral therapy (UNAIDS 2018).

Different behaviors influence or are certainly influenced by another. Multiple risk behaviors among the key population have permitted multiple infections posing more danger among the key population. WHO (2014) report identified overlapping vulnerabilities where people from key population engage in more than one high-risk behavior thus high HIV prevalence rate.

The environment determines how people behave and individual action has an impact on the community and at society at large. Meaning human behavior and environment are interconnected broadening our thinking when addressing the new HIV infection. Involving HIV patients in designing intervention programs will help us understand better the impact of HIV by learning from the affected group to help apply the correct approach in the fight of HIV.

\section{References}

i. AFAO \& Australia's State and Territory AIDS Council (2016). Demonstrating the value of community control in Australia's HIV response. Available at www.afao.org.au Accessed on 24th September 2019.

ii. Allden M. D.K \& Murakami, N. (2015). Trauma and recovery on war's borders. New Hampshire: Dartmouth College Press.

iii. Auerbach, J. Wypijewska, C.\& Brodie, H.K.H. (1994). AIDS and behavior: An Integrated approach. Washington DC: National Academy Press.

iv. Avert (2017). HIV and AIDS in Kenya. Available at www.avert.org. Accessed on 10 $0^{\text {th }}$ September 2018.

v. Avert (2017). Global Information and Education on HIV and AIDS: HIV and AIDS in Kenya. Available at www.avert.org. Accessed on $10^{\text {th }}$ September 2018.

vi. Bowser, P.B. Quimby, E. \& Singer, M. (2007). When communities assess their AIDS Epidemics. New York: Rowman \& Littlefield Publishers Inc.

vii. Camlin, C. et.at. (2016). Men 'missing 'from population-based HIV testing: Insights from qualitative research. Journal of Psychological and Social-Medical Aspects of AIDS/HIV. DOI: 10.1080/09540121.2016.1164806

viii. DeCarlo, A. et al. (2014). Men usually say that HIV testing is for women: Gender dynamics \&perceptions of HIV testing in Lesotho. DOI: 10.1080/13691058.2014.913812

ix. Ferrand, A.R. \& Kranzer, K. (2014). Effect of community-based HIV counselling and testing on HIV incidence. Lancet Global Health. Vol 2 Issues 5. DOI org/10.1016/S2214-109X (14)70204-9.

x. Gitau, T. et al. (2016). A Baseline Study on Child Marriage, teenage pregnancy and female genital mutilation/cutting in Kenya. Amsterdam: Royal Tropical Institute.

xi. Katirayi, L. et.al (2017). Echos of old HIV paradigms: Reassessing the problem of engaging men in HIV testing and treatment through women's perspectives. DOI: 10.1186/s12978-017-0387-1

xii. Kibicho, W. (2009). Sex Tourism in Africa Kenya's booming industry. Ottawa: Routledge.

xiii. Journal of Acquired Immune Deficiency Syndrome (JAIDS), (2018). Finding Hidden HIV Clusters to Support Geographical-Oriental HIV Interventions in Kenya. Vol 78. Issue 2. Available at www.journals.lww.com. Accessed on $5^{\mathrm{TH}}$ October 2018.

xiv. Marlatt, A. G. Lerimer, E. M \& Witkiewitz, K. (2012). Harm Reduction Pragmatic Strategies for Managing High Risk Behaviors. 2ndED. New York: The Guilford Press. 
xv. Management Science for Health (MSH). (2018). Most-At-Risk Populations (MARPS). Available at www.msh.org. Accessed on $10^{\text {th }}$ September 2018.

xvi. Mayer, H.K. \& Pizer, F.H. (2009). HIV prevention: A compressive approach. London: Academic Press.

xvii. Mbete, B. Nougier, M. \& Bridge, J. (2013). HIV Harm Reduction and Drug Policy in Kenya. Available at www.filesever.idpc.net. Accessed on $18^{\text {th }}$ September 2018.

xviii. Ministry of Health (2014). Kenya HIV Prevention Revolution Road Map: Count Down to 2030. Available at www.reliefweb.int. Accessed on $3^{\text {rd }}$ Oct 2018.

xix. Ministry of Health (2018). National Implementation Guidelines for HIV and STI Programming Among Young Key Populations. Available at www.iavi.org .Accessed on 20 $0^{\text {th }}$ October 2018.

xx. Musyoki, H. et al. (2018). Change in HIV Prevention Program Outcomes Among Key Population in Kenya: Data from Periodic Surveys. Available at www.journals.plos.org. Accessed on $8^{\text {th }}$ October 2018.

xxi. Musyoki, H, (2012). An Overview of Programs for People Who Inject Drugs. Available at www.uonbi.ac.ke. Accessed on $5^{\text {th }}$ September 2018.

xxii. NACC. (2018). Kenya HIV Estimate Report 2018. Available at www.nacc.or.ke.Accessed on $20^{\text {th }}$ September 2018.

xxiii. NACC \& NASCOP (2014). Kenya HIV County Profiles. Available at www.nacc.or,ke. Accessed on $20^{\text {th }}$ September 2018.

xxiv. NCBI. (2014). Widow cleansing and inheritance among the Luo in Kenya: the need for additional women-centred HIV prevention options. Journal of the International AIDS Society. Doi: 10.7448/IAS.17.1.19010

xxv. Ngigi, S. \& Busolo, N.D. (2018). Behavior Change Communication in Health Promotion: Appropriate Practices and Promising Approaches. International Journal of Innovative Research and Development. DOI: 10.24940/ijird/2018/v7/i9/SEP18027

xxvi. PMC (2016). Population at Increased Risk for HIV Infection in Kenya: Result from National Population Based Household Survey 2012. Available at www.ncbi.nlm.nih.gov.

xxvii. Population Council (2018). New Prevention Approaches for Most-at-Risk at HIV R4P 2018. Available at www.popcouncil.org. Accessed on $5^{\text {th }}$ Oct 2018.

xxviii. Ramanathan, S.et al. (2017). Mapping and Size Estimation of High-Risk Population in Large Scale HIV Prevention Programs: How Good is Good Enough? Vol 5 Issue 5. Available at www.medcraveonline.com. Accessed on 12 September 2018.

xxix. Ramirez-Valles, J. (2002). The protective effects of community involvement for HIV risk behaviors: A Conceptual framework. Health Education Research. Vol. 17. Issues 4. DOI: 10.1093/HER/17.4.389.

xxx. Rosenberg, E.N. et al. (2019). Assessing the impact of a small-group behavior intervention on sexual behaviors among adolescent girls and young women in Lilongwe Malawi: Aquasi-experimental cohorts' study. Available at www.springer.com. Accessed on $12^{\text {th }}$ Sept 2019. Doi: 10.1007/s10461-019-02669-4

xxxi. Sobo, J. E. (1995). Choosing unsafe sex: AIDS-Risk denial among the disadvantaged women. Philadelphia: University of Pennsylvanian Press.

xxxii. UNAID \& WHO (2010). Guidelines on Estimating the Size of Populations Most at Risk to HIV. Geneva: WHO Press.

xxxiii. UNAIDS \& Stop AIDS Alliance (2015). Communities deliver: The critical role of communities in reaching global targets to end the AIDS epidemic. Available at www.unaids.org. Accessed on 24th September 2019.

xxxiv. UNAIDS (2018). Global HIV \& AIDS statistics - 2018 fact sheet. Available at www.unaids.org. Accessed on $5^{\text {th }}$ October 2018.

xxxv. UNAIDS. (2018). UNAIDS data 2018. Available at www.unaids.org. Accessed on 30th September 2019.

xxxvi. WHO (2004). Comparative quantification of health risks: Global and regional burden of disease attributable to selected major risk factors. Vol I Geneva: Bio Medical Central Ltd

xxxvii. WHO \& UNAIDS (2011). Guidelines on Surveillance among Populations Most at Risk for HIV. Available at www.who.int. Accessed on $5^{\text {th }}$ October 2018

xxxviii. WHO. (2014). Consolidated guidelines on HIV prevention, diagnosis, treatment and care for key population. Geneva: WHO Press.

xxxix. WHO. (2018). Summary of the global HIV epidemic 2018. Available at www.who.int/gho/hiv/en. Accessed on $30^{\text {th }}$ September 2019. 